\section{Microbiological flora dynamics in a vacuum packed ripened cheese after thermal abuse}

\author{
Monica Gramaglia, Alberto Bellio, \\ Guerrino Macori, Daniela Manila Bianchi, \\ Maria Pia Potenza, Sara Astegiano, \\ Fabio Zuccon, Lucia Decastelli \\ Struttura Complessa del Controllo \\ Alimenti e Igiene delle Produzioni, \\ Istituto Zooprofilattico Sperimentale \\ del Piemonte, Liguria e Valle d'Aosta, \\ Torino, Italy
}

\section{Abstract}

Food safety must be guaranteed by the synergic action of all actors in production chain. The consumers cover an important role to ensure good storage conditions according to the manufacturer's instructions. The purpose of this study is to evaluate the influence on microbial parameters of storage temperature during shelf life of sliced vacuum packaged traditional cheese. One wheel of raw milk cheese was produced and ripened for 60 days. The cheese was portioned in 10 slices (200 g) and stored at $+4^{\circ} \mathrm{C}$ for 28 days; then, three of these were stored at $+10^{\circ} \mathrm{C}$ from day 29 to 56 and the others continued the storage at $+4^{\circ} \mathrm{C}$ from day 29 to 56. Analyses on mesophilic aerobic microorganisms, total coliforms, Enterobacteriaceae, mesophilic lactococci, thermophilic lactococci, thermophilic lactobacilli, $\mathrm{pH}$ and $\mathrm{Aw}$ were performed at $7,14,21,28,35,49,56$ days. The aerobic bacteria and lactic flora did not significantly changed during the study; coliforms and Enterobacteriaceae showed a greater reduction in the slices stored at +4 than at $+10^{\circ} \mathrm{C}$. Data confirm the maintenance of the refrigeration temperature as an important tool for reducing the risk of microbiological growth in food.

\section{Introduzione}

La vita commerciale dei prodotti alimentari è condizionata da molteplici fattori, quali ad esempio le caratteristiche dell'alimento, la tipologia di confezionamento e le condizioni di conservazione. La sicurezza alimentare è frutto dell'azione sinergica di tutti gli attori coinvolti nella filiera alimentare. Infatti, in tale contesto non è da sottovalutare il ruolo del consumatore che, una volta acquistato il prodotto, lo deve conservare secondo le indicazioni del produttore. In Europa, Australia, Nuova Zelanda, Stati Uniti e Canada oltre l'87\% delle malattie alimentari ha, infatti, origine a livello domestico (Sharma et al., 2009). In ambiente casalingo, la conservazione dei prodotti deperibili ad una temperatura corretta risulta fondamentale; tuttavia, i dati raccolti nell'ambito di uno studio condotto nell'Italia centro-settentrionale hanno mostrato che nel $70 \%$ dei frigoriferi domestici la temperatura è superiore ai $+4^{\circ} \mathrm{C}$ (Vergara et al., 2010).

Di seguito sono presentati i dati preliminari di uno studio condotto su un formaggio porzionato e confezionato sottovuoto. Scopo del lavoro è valutarne le caratteristiche microbiologiche durante la shelf-life sia in condizioni di conservazione corretta $\left(+4^{\circ} \mathrm{C}\right)$ sia in condizioni di abuso termico $\left(+10^{\circ} \mathrm{C}\right)$.

\section{Materiali e Metodi}

All'interno del caseificio sperimentale della Struttura Complessa del Controllo Alimenti e Igiene delle Produzioni dell'Istituto Zooprofilattico Sperimentale del Piemonte, Liguria e Valle d'Aosta, è stato prodotto un formaggio tipo Toma a base di latte vaccino. Il latte crudo $(50 \mathrm{~L})$ è stato scaldato in caldaia fino a $36^{\circ} \mathrm{C}$; successivamente è stato aggiunto il caglio liquido di vitello secondo le indicazioni del produttore (Caglificio Lerici Spa, Cadorago, Italia). Dopo circa 40 min di sosta è stata eseguita una rottura grossolana della cagliata e si è proceduto alla spinatura fino a $48^{\circ} \mathrm{C}$, continuando progressivamente nella rottura fino ad ottenere le dimensioni di chicco di mais. La cagliata è stata estratta mediante tela e posizionata in fascera. Si è proceduto alla pressatura meccanica per favorire lo spurgo del siero per circa 18 ore con 5 rivoltamenti della forma. La forma in seguito è stata immersa in salamoia al $22 \%$ di $\mathrm{NaCl}$ per 12 ore e successivamente riposta in cella climatica $\mathrm{a}+10^{\circ} \mathrm{C}$ [umidità relativa (U.R.) 90\%] per 60 giorni ottenendo una forma di circa $5 \mathrm{~kg}$, con diametro di $30 \mathrm{~cm}$ e scalzo di circa $15 \mathrm{~cm}$.

Il formaggio è stato porzionato in 10 fette, da circa 200 gr ciascuna, che sono state confezionate sottovuoto $(0,8$ bar) in sacchetti multistrato goffrati per la conservazione degli alimenti, in materiale plastico atossico e inerte; il valore di permeabilità del materiale all'ossigeno risulta essere $50 \mathrm{cc} / \mathrm{m}^{2} / 24 \mathrm{~h}$ (metodo DIN $53380,23^{\circ} \mathrm{C}$ al $75 \%$ U.R.) mentre per l'anidride carbonica è $10 \mathrm{cc} / \mathrm{m}^{2} / 24 \mathrm{~h}$ (metodo DIN 53380, $23^{\circ} \mathrm{C}$ ). Inoltre è stata tagliata un'altra fetta, che non è stata però confezionata, la quale è stata poi utilizzata per le analisi del formaggio al tempo zero, cioè prima del confezionamento sottovuoto. Al fine di riprodurre le condizioni reali di vita commerciale del prodotto, tutte le fette sono state conservate $\mathrm{a}+4^{\circ} \mathrm{C}$ per 28 giorni, simulando un corretto stoccaggio a livello di stabilimento di produzione o di grande distri-
Correspondence: Lucia Decastelli, Struttura Complessa del Controllo Alimenti e Igiene delle Produzioni, Istituto Zooprofilattico Sperimentale del Piemonte, Liguria e Valle d'Aosta, via Bologna 148, 10154 Torino, Italy.

Tel. +39.011 .2686233 - Fax: +39.011 .22473450 .

E-mail: lucia.decastelli@izsto.it

Key words: Shelf-life, Cheese, Vacuum packed, Thermal abuse.

Conflict of interests: the authors declare no potential conflict of interests.

Received for publication: 15 January 2013. Revision received: 21 January 2013.

Accepted for publication: 21 January 2013.

This work is licensed under a Creative Commons Attribution 3.0 License (by-nc 3.0).

(C) Copyright M.Gramaglia et al., 2013

Licensee PAGEPress, Italy

Italian Journal of Food Safety 2013; 2:e10

doi:10.4081/ijfs.2013.e10

buzione organizzata (GD0). Successivamente tre fette sono state riposte $\mathrm{a}+10^{\circ} \mathrm{C}$ a partire dal giorno 29 e fino al giorno 56 , simulando una temperatura scorretta del frigorifero domestico, mentre le restanti tre sono state mantenute $\mathrm{a}+4^{\circ} \mathrm{C}$.

Ad intervalli regolari, e precisamente ai giorni $7,14,21,28,35,49$, 56, sono stati prelevati i campioni, per sottoporli alle seguenti analisi microbiologiche quantitative:

microrganismi mesofili aerobi a $30^{\circ} \mathrm{C}$ - (ISO 4833:2003) (ISO, 2003);

coliformi totali - (ISO 4832:2006) (ISO, 2006);

Enterobacteriaceae - (ISO 21528-2:2004) (ISO, 2004b);

lattococchi mesofili - $\left(\mathrm{M} 17\right.$ agar; $+30^{\circ} \mathrm{C}$ per 48 ore);

lattococchi termofili - (M17 agar; $+45^{\circ} \mathrm{C}$ per 48 ore);

lattobacilli termofili - (MRS agar; $+45^{\circ} \mathrm{C}$ per 72 ore);

Su tutti i campioni, inoltre, sono stati misurati pH (metodo normato MFHPB-03: February 2003) e water activity (Aw) (ISO 21807:2004) (ISO, 2004a).

\section{Risultati}

I risultati microbiologici sono riassunti nella Tabella 1. Al momento del confezionamento (giorno 0) il formaggio presentava una carica batterica di 8,0 Log UFC/g, mentre a fine vita commerciale (giorno 56) i valori erano aumentati fino a 8,4 Log UFC/g e 8,7 Log UFC/g rispettivamente $\mathrm{a}+4 \mathrm{e}+10^{\circ} \mathrm{C}$. I coliformi tota- 
Tabella 1. Risultati delle analisi microbiologiche ( $\left.\log _{10} \mathrm{UFC} / \mathrm{g}\right)$ effettuate sul formaggio porzionato stoccato $\mathrm{a}+4^{\circ} \mathrm{C}$ e a $+10^{\circ} \mathrm{C}$.

\begin{tabular}{cccccc} 
Gioni CMI & $\begin{array}{c}\text { Coliformi } \\
\text { totali }\end{array}$ & $\begin{array}{c}\text { Enterobatte- } \\
\text { riaceae }\end{array}$ & $\begin{array}{c}\text { Lattococchi } \\
\text { mesofili }\end{array}$ & $\begin{array}{c}\text { Lattococchi } \\
\text { termofili }\end{array}$ & $\begin{array}{c}\text { Lattobacilli } \\
\text { mesofili }\end{array}$ \\
\hline$+4^{\circ} \mathrm{C}$ & $+4^{\circ} \mathrm{C}$ & $+4^{\circ} \mathrm{C}$ & $+4^{\circ} \mathrm{C}$ & $+4^{\circ} \mathrm{C}$ & $+4^{\circ} \mathrm{C}$
\end{tabular}

\begin{tabular}{ccccccc}
0 & 8,0 & 5,1 & 4,8 & 7,0 & 8,4 & 7,7 \\
7 & 8,2 & 3,5 & 4,2 & 8,1 & 8,0 & 7,1 \\
\hline 14 & 8,3 & 4,1 & 4,2 & 7,7 & 8,1 & 7,0 \\
21 & 8,6 & 4,1 & 4,1 & 6,8 & 7,2 & 7,6 \\
\hline 28 & 8,0 & 4,0 & 3,7 & 7,3 & 8,1 & 7,1 \\
& $+4^{\circ} \mathrm{C}+10^{\circ} \mathrm{C}+4^{\circ} \mathrm{C}+10^{\circ} \mathrm{C}$ & $+4^{\circ} \mathrm{C}+10^{\circ} \mathrm{C}$ & $+4^{\circ} \mathrm{C}+10^{\circ} \mathrm{C}$ & $+4^{\circ} \mathrm{C}+10^{\circ} \mathrm{C}$ & $+4^{\circ} \mathrm{C}+10^{\circ} \mathrm{C}$
\end{tabular}

\begin{tabular}{lllllllllllll}
35 & 8,4 & 8,4 & 3,8 & 3,9 & 3,3 & 4,2 & 8,0 & 8,1 & 8,1 & 8,1 & 7,6 & 7,6 \\
49 & 8,3 & 8,6 & 3,0 & 3,2 & 3,8 & 3,9 & 7,9 & 8,4 & 8,1 & 8,3 & 7,1 & 7,7 \\
\hline 56 & 8,4 & 8,7 & 2,6 & 3,9 & 3,2 & 4,0 & 8,1 & 8,0 & 8,1 & 8,1 & 8,1 & 6,7 \\
\hline
\end{tabular}

CMT, Carica mesofila totale.

li (5,1 Log UFC/g al giorno 0) hanno mostrato un trend negativo durante la prima settimana a $+4^{\circ} \mathrm{C}$; nelle settimane successive questo trend si è conservato solo per le porzioni mantenute refrigerate. Le concentrazioni finali sono rispettivamente di $2,6 \mathrm{Log} \mathrm{UFC} / \mathrm{g}\left(+4^{\circ} \mathrm{C}\right)$ e $3,9 \mathrm{Log} \mathrm{UFC} / \mathrm{g}\left(+10^{\circ} \mathrm{C}\right)$. Il conteggio delle Enterobacteriaceae è risultato pressoché costante dal giorno 0 al giorno 28 (conservazione $\left.\mathrm{a}+4^{\circ} \mathrm{C}\right)$; in seguito tale valore è diminuito progressivamente solo nei campioni mantenuti a $+4^{\circ} \mathrm{C}(3,2 \mathrm{Log} \mathrm{UFC} / \mathrm{g}$ al giorno $56)$. La flora lattica non ha mostrato variazioni significative durante il periodo di studio mantenendosi compresa tra 7,0 e 8,0 Log UFC/g.

Il $\mathrm{pH}$ è rimasto compreso tra 5,5 e 5,3 in entrambe le condizioni di stoccaggio, mentre l'Aw è diminuito da 0,97 - valore del formaggio al giorno 0 - a 0,90 del giorno 7, rimanendo poi pressoché costante con valori compresi tra 0,90 e $0,92\left(+4^{\circ} \mathrm{C}\right)$ e 0,92 e 0,91 al giorno 56 $\left(+10^{\circ} \mathrm{C}\right)$ (Tabella 2$)$.

\section{Discussione}

Il confezionamento sottovuoto di formaggi porzionati è una pratica molto impiegata negli ultimi anni soprattutto a livello di GD0: il prodotto conserva le proprie caratteristiche organolettiche, è facilmente conservabile a livello domestico e il suo periodo di shelf-life può essere prolungato. Tuttavia, per diverse tipologie di alimenti, il confezionamento sottovuoto non prescinde dal mantenimento della catena del freddo e dalla conservazione del prodotto a temperatura di refrigerazione sia a livello di GD0 che a livello domestico dopo l'acquisto.

Questo studio ha infatti rilevato un gradua- le aumento della carica mesofila totale nelle confezioni mantenute a $+10^{\circ} \mathrm{C}$; i coliformi totali e le Enterobacteriaceae hanno seguito un trend negativo nelle porzioni mantenute a $+4^{\circ} \mathrm{C}$, ma la riduzione delle loro concentrazioni ha subito un rallentamento quando si è verificato l'abuso termico. Le basse temperature e la riduzione, parziale o totale, dell'ossigeno inibiscono la proliferazione di batteri Gram negativi che frequentemente contaminano gli alimenti (Borch et al., 1996; Pintado and Malcata, 2000; Nychas et al., 2008).

La flora lattica non ha mostrato variazioni significative durante il periodo di studio, e anche i valori di pH e Aw sono rimasti costanti. I batteri Gram positivi, rappresentati in questo studio dalla flora lattica, sono poco sensibili alla riduzione 0 alla mancanza d'ossigeno (Björkroth et al., 2005; Abadias et al., 2008; Gammariello et al., 2009).

\section{Conclusioni}

Nonostante alcuni limiti metodologici riguardanti eventuali bias legati alla possibile variabilità dei dati ottenuti in assenza di replicati delle analisi, i risultati preliminari di questo studio mostrano che gli abusi termici, a cui un prodotto lattiero-caseario stagionato può essere sottoposto durante la sua vita commerciale, possono compartecipare, insieme ad altri fattori, alla modifica delle concentrazioni della popolazione batterica. Il lavoro suggerisce che il confezionamento sottovuoto, unito ad un corretto mantenimento della temperatura di refrigerazione, è in grado di mantenere inalterate le caratteristiche microbiologiche del formaggio oggetto del lavoro.
Tabella 2. Risultati delle analisi chimicofisiche effettuate sul formaggio porzionato stoccato $\mathrm{a}+4^{\circ} \mathrm{C}$ e a $+10^{\circ} \mathrm{C}$.

\begin{tabular}{lcccc} 
Giomi & \multicolumn{2}{c}{$\mathrm{pH}$} & \multicolumn{2}{c}{$\mathrm{Aw}$} \\
& $+4^{\circ} \mathrm{C}$ & $+10^{\circ} \mathrm{C}$ & $+4^{\circ} \mathrm{C}$ & $+10^{\circ} \mathrm{C}$ \\
0 & 5,5 & - & 0,97 & - \\
7 & 5,5 & - & 0,90 & - \\
\hline 14 & 5,3 & - & 0,92 & - \\
21 & 5,4 & - & 0,93 & - \\
\hline 28 & 5,3 & - & 0,92 & - \\
35 & 5,4 & 5,4 & 0,91 & 0,92 \\
\hline 49 & 5,3 & 5,4 & 0,91 & 0,90 \\
56 & 5,3 & 5,3 & 0,92 & 0,91 \\
\hline
\end{tabular}

AW, water activity.

\section{Bibliografia}

Abadias M, Usall J, Anguera M, Solsona C, Viñas I, 2008. Microbiological quality of fresh, minimally-processed fruit and vegetables, and sprouts from retail establishments. Int J Food Microbiol 123:121-9.

Björkroth KJ, Ristiniemi M, Vandamme P, Korkeala JH, 2005. Enterococcus species dominating in fresh modified-atmospherepackaged, marinated broiler legs are overgrown by Carnobacterium and Lactobacillus species during storage at $6^{\circ} \mathrm{C}$. Int J Food Microbiol 97:267-76.

Borch E, Kant-Muermans ML, Blixt Y, 1996. Bacterial spoilage of meat and cured meat products. Int J Food Microbiol 33:103-20.

Gammariello D, Conte A, Di Giulio S, Attanasio A, Del Nobile MA, 2009. Shelf life of stracciatella cheese under modified-atmosphere packaging. J Dairy Sci 92:483-90.

ISO, 2003. Microbiology of food and animal feeding stuffs. Horizontal method for the enumeration of microorganisms. Colonycount technique at 30 degrees C. Norma ISO 4833:2003. Organizzazione internazionale per la normazione ed., Ginevra, Svizzera.

ISO, 2004a. Microbiology of food and animal feeding stuffs. Determination of water activity. Norma ISO 21807:2004. Organizzazione internazionale per la normazione ed., Ginevra, Svizzera.

ISO, 2004b. Microbiology of food and animal feeding stuffs. Horizontal methods for the detection and enumeration of Enterobacteriaceae. Part 2: colony-count method. Norma ISO 21528-2:2004. Organizzazione internazionale per la nor- 
mazione ed., Ginevra, Svizzera.

ISO, 2006. Microbiology of food and animal feeding stuffs. Horizontal method for the enumeration of coliforms. Colony-count technique. Norma ISO 4832:2006. Organizzazione internazionale per la normazione ed., Ginevra, Svizzera.

Nychas G-J, Skandamis P, Tassou C,
Koutsoumanis K, 2008. Meat spoilage during distribution. Meat Sci 78:77-89.

Pintado ME, Malcata FX, 2000. Characterization of whey cheese packaged under vacuum. J Food Protect 63:216-21.

Sharma M, Eastridge J, Mudd C, 2009. Effective household disinfection methods of kitchen sponges. Food Control 20:310-3.
Vergara A, Festino AR, Di Ciccio P, Pennisi L, Conter M, Costanzo C, Ianieri A, 2010. La gestione della refrigerazione domestica: caratteristiche igienico-sanitaria di frigoriferi dell'Italia centro-settentrionale. Rivista della Associazione Italiana Veterinari Igienisti 8:13-7. 